\title{
More flexibility for code generation with GeNN v2.1
}

\author{
Thomas Nowotny*, James Turner, Esin Yavuz \\ From 24th Annual Computational Neuroscience Meeting: CNS*2015 \\ Prague, Czech Republic. 18-23 July 2015
}

\section{Background}

GeNN (GPU enhanced Neuronal Networks) [1,2] is a software framework that was designed to facilitate the use of GPUs (Graphics Processing Units) for the simulation of spiking neuronal networks. It is built on top of the CUDA (Common Unified Device Architecture) [3] application programming interface provided by NVIDIA Corporation and is entirely based on code generation: Users provide a compact description of a spiking neuronal network model and GeNN generates CUDA and $\mathrm{C}++$ code to simulate it, also taking into account the specifics of the GPU hardware detected at compile time.

\section{Methods}

In this contribution we describe novel work on GeNN, which has transformed it to a yet more flexible tool for facilitating the use of GPUs for simulations accelerated by GPUs. The main innovations involve replacing previous fixed templates for synapse dynamics and learning models by user-definable code snippets, so allowing redefinition of virtually every dynamic element of a neural network simulation. This transition has also enabled the completion of the Brian2 to GeNN and SpineML to GeNN interfaces [4].

\section{Results}

GeNN now allows the free definition of all four, neuron dynamics, neuron threshold conditions, synapse dynamics and connection weight dynamics (learning). The desired behavior is encoded in code snippets that contain $\mathrm{C}++$ compatible code that describes the operations that are necessary to complete one time step. Table 1 summarizes the available code slots and their function.

Other improvements in GeNN 2.1 include an improved CUDA block size estimation algorithm, access to pre- and post-synaptic variables in synaptic models, and a number of bug fixes.

\section{Conclusion}

GeNN has reached level of stability where it should be of increasing use to the wider computational neu-

Table 1 Summary of code slots available in GeNN for user-defined models

\begin{tabular}{lll}
\hline Element & Snippet & Deployment and Function \\
\hline seuron & simCode & Main time step update of the neuron dynamics \\
\hline thresholdConditionCode & A Boolean expression defining when spikes occur, checked every time step \\
\hline resetCode & The code that defines a change in neuron variables, employed when a spike occurs \\
\hline simCode & Code that describes the synapse update after a pre-synaptic spike \\
\hline simCodeEvnt & Code that describes the synapse update after a pre-synaptic spike event \\
\hline sost-Synapse & eventThreshold & Code for the synaptic update triggered by a post-synaptic spike \\
\hline & postSyntoCurrent & A Boolean expression that defines synaptic events \\
\hline
\end{tabular}

* Correspondence: t.nowotny@sussex.ac.uk

CCNR, School of Engineering and Informatics, University of Sussex, Falmer,

Brighton BN1 9QJ, UK 
roscience community, in particular with the completion of its interfaces to other simulators.

\section{Acknowledgements}

This work was supported by the EPSRC (Green Brain Project, grant number EP/J019690/1) and a Royal Academy of Engineering/Leverhulme Trust

Fellowship.

Published: 18 December 2015

\section{References}

1. Nowotny T: Flexible neuronal network simulation framework using code generation for NVidia ${ }^{\oplus}$ CUDA $^{\mathrm{T}}{ }^{\mathrm{N}}$. BMC Neuroscience 2011, 12(Suppl 1):P239.

2. Yavuz E, Turner J, Nowotny T: Simulating spiking neural networks on massively parallel graphical processing units using a code generation approach with GeNN. BMC Neuroscience 2014, 15(Suppl 1):01.

3. CUDA. [http://www.nvidia.com/object/cuda_home_new.html], accessed 2015-02-25.

4. Nowotny T, Cope AJ, Yavuz E, Stimberg M, Goodman DFM, Marshall J, Gurney K: SpineML and Brian 2.0 interfaces for using GPU enhanced Neuronal Networks (GeNN). BMC Neuroscience 2014, 15(Suppl 1):P148.

doi:10.1186/1471-2202-16-S1-P291

Cite this article as: Nowotny et al:: More flexibility for code generation with GeNN v2.1. BMC Neuroscience 2015 16(Suppl 1):P291.

\section{Submit your next manuscript to BioMed Central} and take full advantage of:

- Convenient online submission

- Thorough peer review

- No space constraints or color figure charges

- Immediate publication on acceptance

- Inclusion in PubMed, CAS, Scopus and Google Scholar

- Research which is freely available for redistribution

Submit your manuscript at www.biomedcentral.com/submit 